\title{
Optimization of Povidone K-30 and Sodium Starch Glycolate on Levofloxacin Tablet by Factorial Design
}

\author{
Widji Soeratri ${ }^{1}$, Mahrus Naufal Nuruddin ${ }^{1}$, Diajeng Putri Paramita ${ }^{1}$, \\ Dwi Setyawan $^{1 *}$, Bambang Widjaja ${ }^{2}$ \\ ${ }^{I}$ Department of Pharmaceutics, Faculty of Pharmacy, Universitas Airlangga \\ ${ }^{2}$ Department of Pharmacy, Medical Faculty, Universitas Hang Tua, \\ *E-mail: dwisetyawan-90@ff.unair.ac.id
}

\begin{abstract}
The aim of this study was to determine the effect of binder and disintegrant excipients toward tablet properties of levofloxacin as the latter tends to suffer brittle fracture upon compression. The excipients used were povidone K-30 as the binder and sodium starch glycolate (SSG) as the disintegrant which the tablets were formulated according to factorial design $2^{2}$ with two factors and two levels on each factor. Four formulas were prepared by wet granulation method using 2 and $4 \%$ of each povidone K-30 and sodium starch glycolate in various compositions. Tablet properties were evaluated for its hardness, friability, and disintegration time as well as dissolution profile. The data obtained was statistically analyzed using Minitab ${ }^{\circledR} 17$ software to optimize the formulation and resulted in different impacts caused by each excipient. Povidone K-30 exhibited an increment in hardness, friability, disintegration time but a decrease indissolution profile of levofloxacin tablet. SSG decreased hardnessand disintegration time, but increased friability and dissolution profile of levofloxacin tablet. Overlaid contour plot showed that the optimal formula regarding tablet properties of friability, disintegration time, and dissolution profile is in composition of $2.01 \%$ povidone K-30 and $2.01 \%$ sodium starch glycolate.
\end{abstract}

Keywords: levofloxacin tablet, povidone K-30, sodium starch glycolate, factorial design.

\section{INTRODUCTION}

Commonly used pharmaceutical dosage form is a tablet and it is available in diverse shapes for oral purpose (Setyawan et al., 2013). Tablet is solid dosage form which contains active pharmaceutical ingredient(s) (APIs) with or without excipient. It is produced by compression with high pressure on powder or granulated mass on a die (Ministry of Health of the Republic of Indonesia, 2014). Tablet properties, such as compressibility, friability, and disintegration time, are influenced by characteristic of excipients and tableting process. Excipients such as binder and disintegration agent can improve tablet manufacturing process to meet required specification (Fatmawati et al., 2017). During compression, both API(s) and excipient(s) can undergo brittle fracture. Brittle fracture is direct consequence of stress released from die wall, tablet powder with low density, and fracture center during decompression. Particles with plastic behavior in tablet powder can absorb stress and improve brittle fracture (Uhumwangho \& Okor, 2004).

Levofloxacin is a synthetic, thirdgeneration fluoroquinolone antibiotic agent with broad-spectrum antibacterial activity.
Levofloxacin is used to treat various infections such as community-acquired pneumonia, acute exacerbation of chronic bronchitis, acute maxillary sinusitis, skin and skin structure infection, urinary infection, and acute pyelonephritis (Nazir et al., 2013). Levofloxacin is also known to be slightly soluble in water and tends to suffer brittle fracture that can impact its formulation and dissolution. Levofloxacin is susceptible to break (fragmented) so that it needs binder to improve its physical quality and dissolution rate (Fatmawati et al., 2017).

Polyvinylpyrrolidone, also known as povidone, is normally used as a binder in wet granulation process. It is classified to several types according to its viscosity in aqueous solution that is called as $\mathrm{K}$-value within range of 10-120. Wet granulation using povidone K30 has characterized to produce harder granules with better flowability dan lower friability (Rowe et al., 2009). A correct amount of binder existed in tablet powder can improve plasticity and increase interparticulate bonding. Therefore, tablet compactibility is increased and tendency for brittle fracture (capping or laminating) can be eliminated (Uhumwangho et al., 2006). 
However, increasing binder composition can impact tablet disintegration time significantly as disintegration takes longer time to finish (Chime et al., 2012). To overcome this problem, disintegration agent (abbreviated disintegrant) can be added during process. Disintegrant has major function to reduce the effect of binder and physical treatment during compaction. If high binder composition is present, one should use more effective disintegrant to release API(s) from the tablet form (Mohanachandran et al., 2011).

Superdisintegrant, a new class of disintegrant, is introduced to be more effective and efficient as it possesses greater disintegration effect and mechanical strength (Mohanachandran et al., 2011).

Superdisintegrant acts by absorbing water then swelling to generate pressure outward and cause a tablet to break and disintegrate (Shakar et al., 2012). Sodium starch glycolate (SSG) is one of superdisintegrant which can be used both in direct compression and wet granulation method (Rowe et al., 2009).

Based on aforementioned background, this research was aimed to determine the effect of povidone K-30 as binder and SSG as disintegranton levofloxacin tablet prepared using wet granulation method. Composition of povidone K-30 and SSG was varied in each formula based on factorial design $2^{2}$.

Parameters evaluated included hardness, friability, disintegration time, and dissolution profile. All data obtained then were analyzed with Minitab ${ }^{\circledR}$ software to depict tablet's quality profile and predict the optimum formulation.

\section{METHODS}

\section{Materials}

Materials used in this research were levofloxacin (Shanghai Terpon Chemical Co., LTD., China), povidone K-30 (Sigma Aldrich Co., USA), sodium starch glycolate (Yung ZIP Chemical Co., China), microcrystalline cellulose (Avicel ${ }^{\circledR}$ PH-101, FMC Co., USA), and pharmaceutical grade of corn starch, magnesium stearate, and talc.

\section{Formulation}

Formulation was designed according to factorial design $2^{2}$ by two factors on two levels for tablet containing $500 \mathrm{mg}$ of levofloxacin. The factors were povidone K-30 and SSG at two levels of 2 and 4\% as low andhigh concentration, respectively. Hence, four formulas were obtained which the compositions were represented in Table 1. Formula 1 (F1) consisted of povidone K-30 and SSG in lower concentration; F2 consisted of povidone K-30 and SSG in lower and higher concentration,respectively F3 consisted of povidone K-30 and SSG in higher and lower concentration, respectively. F4 consisted of povidone K-30 and SSG in higher concentration. Tablets were prepared by wet granulation method with aquadest as solvent added. Granule was dried at temperature of $50-60^{\circ} \mathrm{C}$ and sieved through mesh No. 14-20. Tableting was performed using $13 \mathrm{~mm}$ die on tablet hydraulic press by pressure of $9.81 \mathrm{kN}$. Then, the tablet was evaluated for its properties by parameters of hardness, friability, disintegration time, and also in vitro dissolution.

Table 1. Formulations of levofloxacin tablet (mg)

\begin{tabular}{|c|c|c|c|c|c|}
\hline Compounds & Function & F1 & F2 & F3 & F4 \\
\hline Levofloxacin & API & 500 & 500 & 500 & 500 \\
\hline Avicel ${ }^{\circledR}$ PH-101 & Filler & 60 & 60 & 60 & 60 \\
\hline Corn Starch & Filler & 40 & 40 & 40 & 40 \\
\hline Povidone K-30 & Binder & 12.73 & 13.00 & 26.02 & 26.59 \\
\hline SSG & Disintegrant & 12.73 & 26.02 & 13.00 & 26.59 \\
\hline Talk & Glidant & 6.36 & 6.50 & 6.50 & 6.65 \\
\hline Magnesium stearate & Lubricant & 4.77 & 4.88 & 4.88 & 4.98 \\
\hline Aquadest & Solvent & 16 & 16 & 16 & 16 \\
\hline \multicolumn{2}{|c|}{ Total } & 636.59 & 650.40 & 650.40 & 664.81 \\
\hline
\end{tabular}

note: F1 (Formula 1); F2 (Formula 2); F3 (Formula 3); F4 (Formula 4).

\section{Evaluation of Tablet Properties}

Tablet hardness, friability, and disintegration time were measured in three replications to depict tablet properties. The hardness test was performed using aErweka hardness tester TBH 220 (Germany) upon five tablets from each formula. The tablet hardness was measured in kilogram-force or kilopond $(\mathrm{kP})$.

Value between 4-8 $\mathrm{kP}$ is considered to be acceptable (Fatmawati et al., 2017). The friability test was carried out on Erweka friability tester TAP
31914 (Germany). A number of tablets weighed around $6.5 \mathrm{~g}$ was rotated at $25 \mathrm{rpm}$ for 4 minutes. The fines were removed first before re-weighing, then weight loss percentage was calculated. The result is acceptable if tablet friability values less than 1\% (Türkmen et al., 2018).

The disintegration time was determined with Erweka disintegrator ZT 501 (Germany) which was containing a basket rack assembly with six wireended tubes. One tablet was placed in each of six 
tubes, followed by a cover disk, then the basket was raised and lowered in medium of $900 \mathrm{~mL}$ distilled water attemperature of $37 \pm 2^{\circ} \mathrm{C}$. Disintegration is considered to be complete when all tablet fragments pass through the mesh and acceptable time is below 15 minutes for uncoated tablet (Schmid \& Löbenberg, 2010).

\section{In Vitro Dissolution Test}

In vitro dissolution test was conductedusing Erweka DT 700 (Erweka, Germany) with basket apparatus in three replicates for each formula. Dissolution medium used $0.1 \mathrm{~N} \mathrm{HCl}$ in aqueous solution. Each tablet of formula 1-4 was added into $900 \mathrm{~mL}$ dissolution medium and stirred at speed of $100 \mathrm{rpm}$ and temperature of $37 \pm 0.5{ }^{\circ} \mathrm{C}$. About $5.0 \mathrm{~mL}$ of dissolution sample was taken at interval of 10, 20, 30 , dan 45 minutes then filtered through millipore of $0.45 \mathrm{~m}$ diameter. The filtrate was observed on UVVis spectrophotometer (Hitachi UH5300, Japan) at maximum wavelength of levofloxacin $(\lambda=294.98$ $\mathrm{nm})$. Obtained absorbance was used to calculate dissolved levofloxacin using a standard calibration curve. Each sample was analyzed in triplicate.

\section{Statistical Analysis}

The resulting data were analyzed by one-way analysis of variance (ANOVA) using SPSS 21.0 software (SPSS Inc., USA) with significance limit was set at alpha $<0.05$. Minitab $^{\circledR} 17$ (Minitab Inc., Australia) software was used to determine the optimum formula for levofloxacin tablet from hardness, friability, and disintegration time data.

\section{RESULTS AND DISCUSSION}

Levofloxacin is known to possess poor tabletability due to its brittleness. It tends to suffer brittle fracture during compaction even with commonly used wet granulation formula (Fatmawati et al., 2017). Adding excipients with plastic properties is believed to be able to overcome brittle fracture issue (Uhumwangho
\& Okor, 2004). Therefore, in this study, we suggested alternative formulation for levofloxacin tablet that was prepared using wet granulation method. Factorial design was used to construct our experiment with two factors was analyzed for the effect. The factors were povidone K-30 and SSG as those excipients has been known to affect the properties of levofloxacin tablet.The level was determined at 2 and $4 \%$ because it was within concentration range that allowed in a formulation with specified function of excipient used (Rowe et al., 2009).

Factorial design is the design of choice when experiment is purposed to elucidate the effect of different factors and conditions on the result. Its application on pharmaceutical industry is very useful as the effects of several factors (A, B, C) and their interactions (ab, bc, ac, abc) can be determined simultaneously (Genc \& Jalvand, 2008), in this case, with Minitab ${ }^{\circledR} 17$ software. The evaluated properties of levofloxacin tablet are presentedin Table 2. Four formulas exhibited tablet hardness in similar value with the lowest one is F3.

However, none these formulas meethe requirement for tablet hardness (within 4-8 kP range). Statistical analysis showed insignificant differences amongst formulas. Those phenomena might be influenced by the type of disintegrant used in this study. Previous study conducted by Prasad \& Duraivel (2012) showed that using sodium starch glycolate $\left(\right.$ Explotab $^{\circledR}$ ) can increase tablet hardness of glimepiride. Furthermore, Explotab ${ }^{\circledR}$ is capable to generate pellets during wet granulation that can bind particle when it is dried. Our result was in agreement with that reference.

Table 2. Result of Tablet Properties Evaluation

\begin{tabular}{lcccc}
\hline \multicolumn{1}{c}{ Evaluation } & F1 & F2 & F3 & F4 \\
\hline Hardness (kP) & $17.49 \pm 0.03$ & $17.49 \pm 0.03$ & $16.56 \pm 2.07$ & $\begin{array}{c}17.48 \pm \\
\text { Friability (\%) }\end{array}$ \\
$\begin{array}{l}\text { Disintegration Time } \\
\text { (minutes) }\end{array}$ & $1.35 \pm 0.16$ & $1.30 \pm 0.06$ & $1.27 \pm 0.37$ & $0.88 \pm 0.01$ \\
\hline
\end{tabular}

note: F1 (Formula 1); F2 (Formula 2); F3 (Formula 3); F4 (Formula 4).

The friability test resulted in the similar percentage of weight loss for all formulas. Threeout of four formulas showed weight loss greater than $1 \%$ and only $\mathrm{F} 4$ that within the criteria requirement. Statistical analysis also indicated that each formula differs insignificantly $(\alpha>0.05)$. This result might be due to factor of disintegrant type. A study with starch pellets and Explotab ${ }^{\circledR}$ produced tablet with higher friability than other disintegrant (Mehta et al., 2011).

In the disintegration test, it was observed 
that tablet disintegrated completely less than 10 minutes in four formulas. The result complies to United States Pharmacopeia (USP) criteria that disintegration time is less than 15 minutes (Schmid \& Löbenberg, 2010). Every formula demonstrates statistically significant differences $(\alpha<0.05)$.

The dissolution profiles are depictedin Figure 1. Each formula has specific profile, responding to different composition of povidone K-30 and SSG. Percentage ofdissolved levofloxacin for F1, F2, F3, and F4 after45 minutes dissolution test was $93.87 \pm$ $0.67,89.69 \pm 0.60,105.06 \pm 1.40$, and $90.68 \pm$
$0.58 \%$, respectively. This result was used for further statistical analysis both with ANOVA and Minitab ${ }^{\circledR}$ software due to compendia requirement for levofloxacin in tablet dosage form. dissolution rate at 45 minutes.

Specification of levofloxacin tablet refers to the United States Pharmacopeia and National Formulary (USP-NF) which is declared that concentration of levofloxacin should be more than $80 \%$ at 45 minutes (The USP Convention, 2011), so that all formulas meet the requirement. Statistical analysis exhibited significant differences $(\alpha<0.05)$.

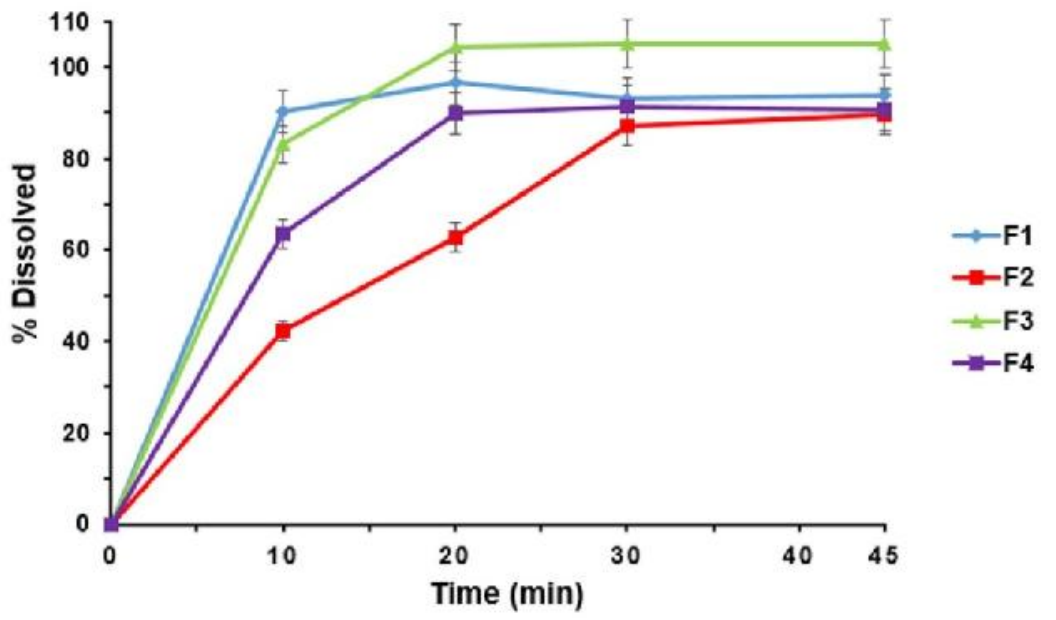

Figure 1. Dissolution profile of levofloxacin tablets in $0.1 \mathrm{~N} \mathrm{HCl}$ at $37 \pm 0.5{ }^{\circ} \mathrm{C}$.

F1 (Formula 1); F2 (Formula 2); F3 (Formula 3); F4 (Formula 4).

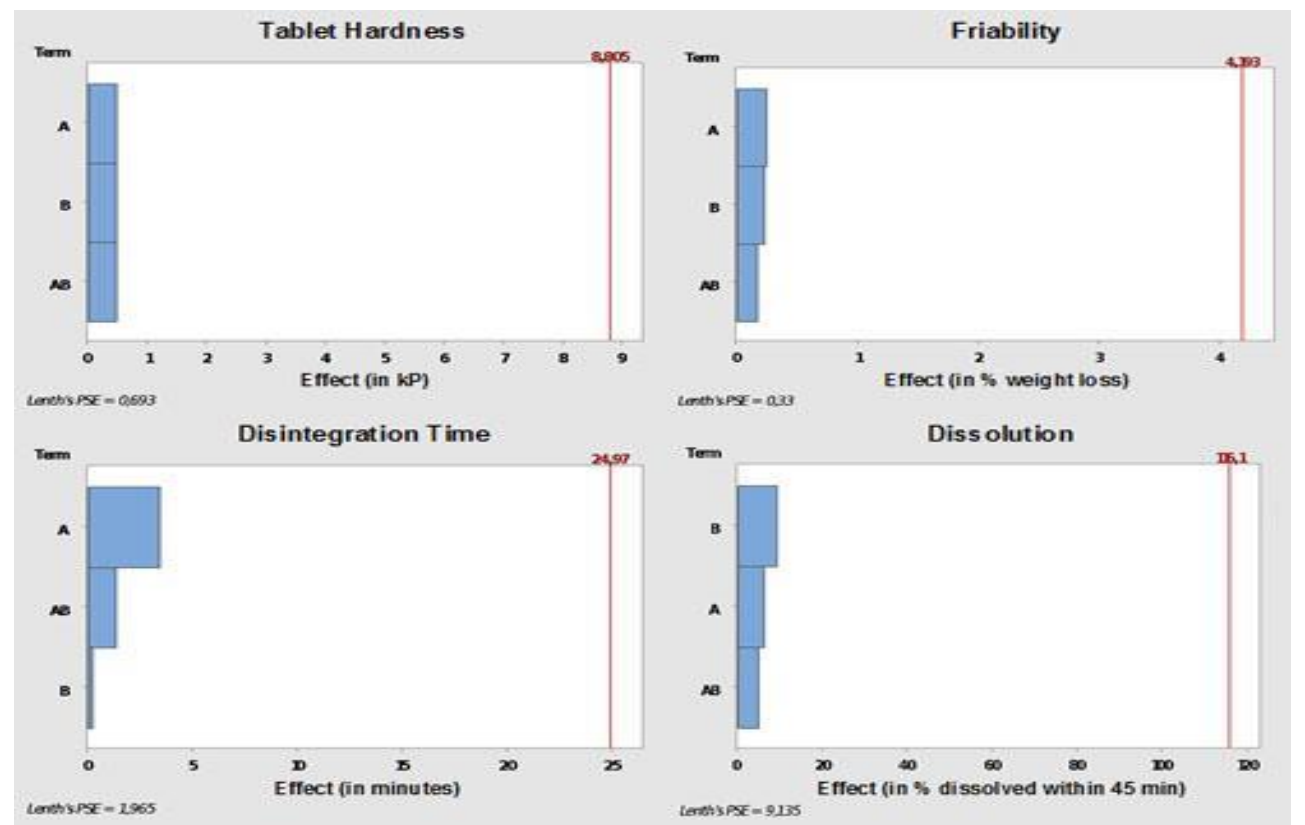

Figure 2. Pareto charts of the effect of povidone K-30 (A) dan SSG (B) as individual factor or in combination $(\mathrm{AB})$ toward levofloxacin tablet properties. 
Statistical analysis was further carried out using Minitab® 17 software to determine the effect of the excipients used by graphical presentation. Pareto chart is used to measure statistically the effect of adding povidone K-30 and SSG on tablet hardness, friability, disintegration time, and dissolution and it is given in Figure 2. A chart that crosses the red line is assumed to have significant effect on the parameter calculated. However, as it can be seen in Figure 2, no graphic crosses the red line. Thus, pareto chart analysis indicatesthat povidone K-30 and SSG do not have major impact on tablet properties and dissolution of levofloxacin tablet.

Another graphic obtained from Minitab ${ }^{\circledR}$ 17 was main effect plot which shows the impact of different level in concentration of povidone K-30 and SSG. Figure 3 shows that an increase in tablet hardness, friability, and disintegration time occurs as concentration of povidone K-30 increases, however a decrease inpercentage dissolved is observed during 45 min dissolution. It also shows that higher level of SSG decreases tablet hardness and disintegration time yet increases friability and dissolution of the tablet.
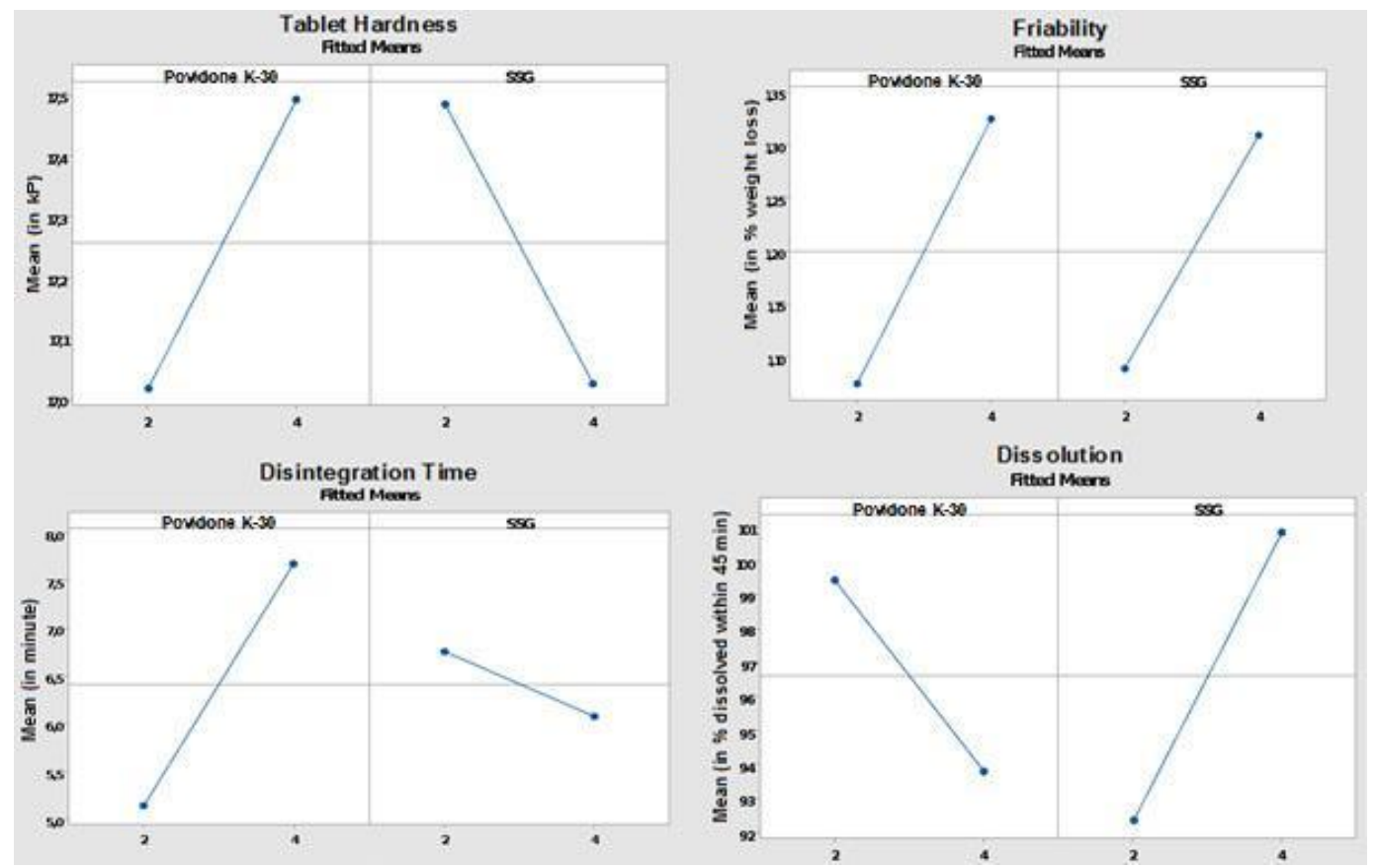

Figure 3. Main effect charts of the effect of povidone K-30 and SSG in two levels toward levofloxacin tablet properties (-1, low level; +1, high level).

Contour plot was also obtained (Figure 4) and it is used to see 3D relationship between povidone K-30 and SSG on levofloxacin tablet properties and dissolution rate. Darker zone represents higher effect on the test conducted. In hardness testing, low concentration of SSG followed by povidone K-30 level increment can increase tablet hardness. Combination of high povidone K-30 concentration with higher concentration of SSG will raise tablet friability. As for disintegration time, it takes longer on increasing level of povidone K-30 in high SSG concentration. Dissolution is enhanced in low povidone K-30 and higher SSG concentration.

If those contour plots are overlaid together, it will helpus to predict the optimum formula to manufacture levofloxacin tablet using povidone K-30 and SSG as it can be seen in Figure 5. The overlay excludes hardness data due to the mismatch of the result to the requirement. White zone on the graphic is the feasible area, thus, the optimum composition of povidone K30 and SSG for levofloxacin tablet formulation are 2.01 and $2.01 \%$ respectively. 

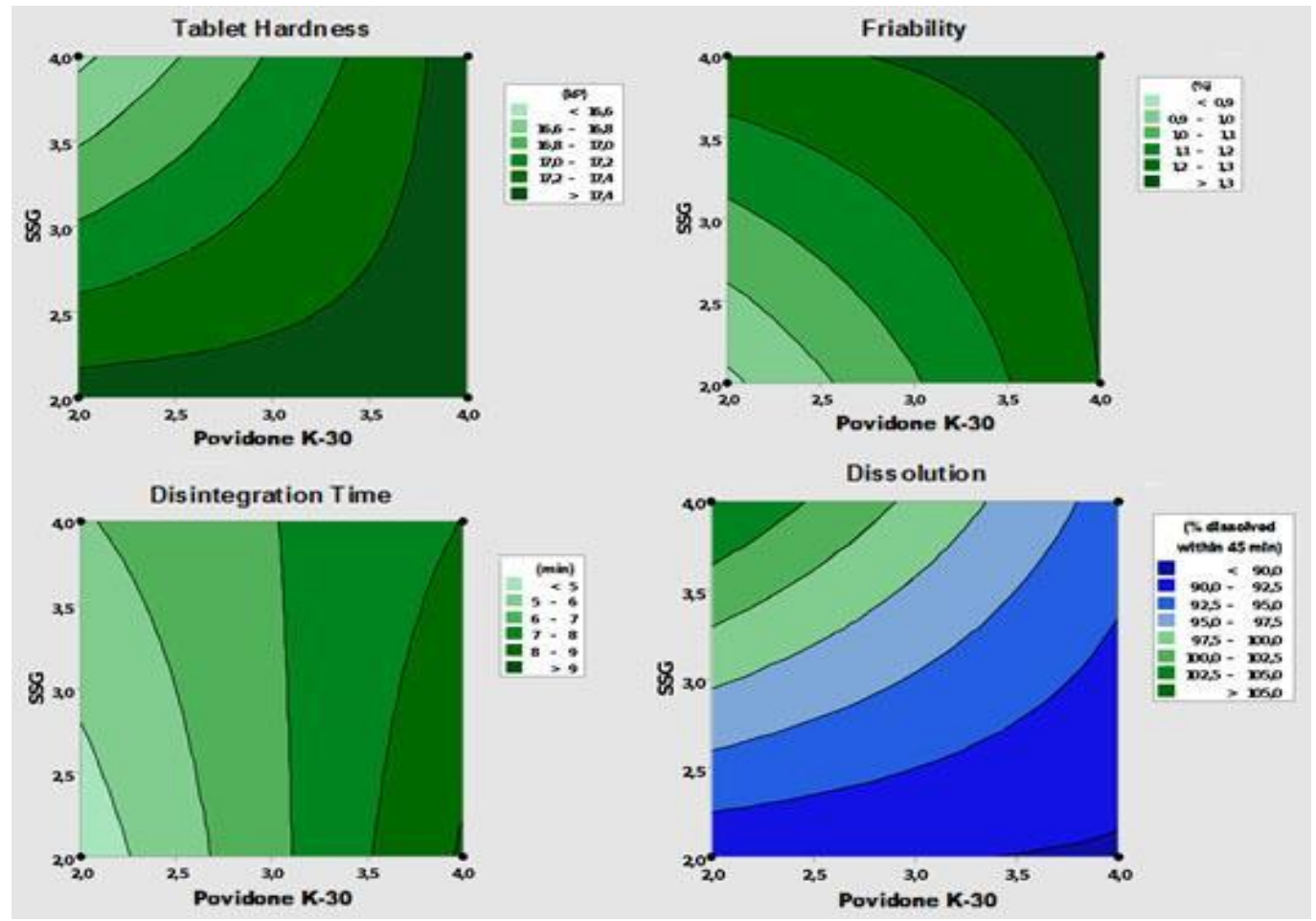

Figure 4. Contour plots of the effect of povidone K-30 and SSG in two level on levofloxac in tablet properties (2.0, low level; 4.0, high level).

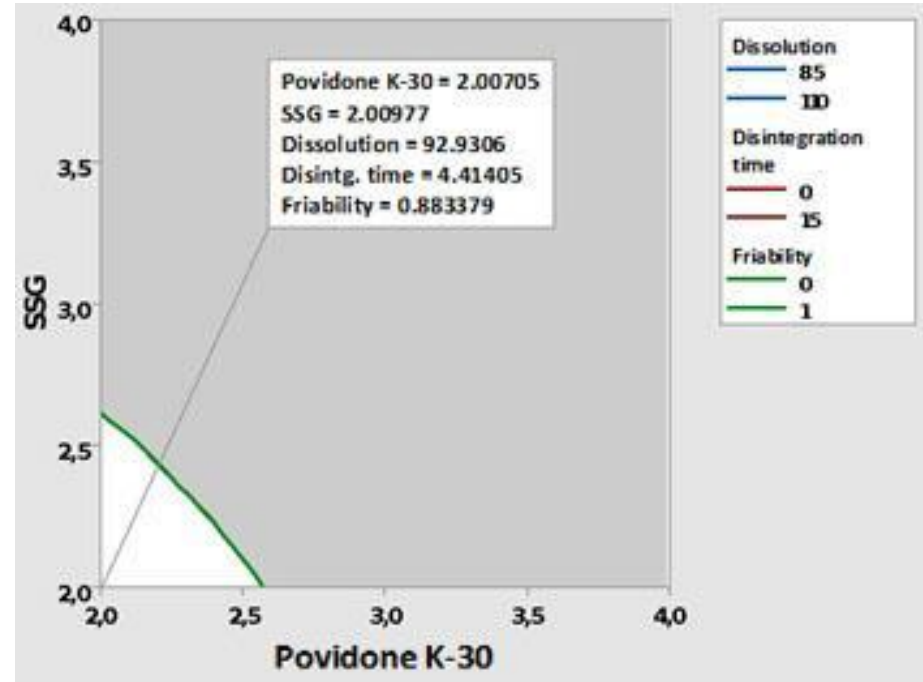

Figure 5. Overlaid contour plot of tablet hardness, friability, and dissolution to determine the optimum formula for levofloxacin tablet.

\section{CONCLUSION}

Povidone K-30 and sodium starch glycolate (SSG) were used in this research to improve levofloxacin brittleness and were proved to impact tablet properties and dissolution rate. Povidone K-30 increases tablet hardness and friability, lengthens disintegration time, but reduces dissolution rate. In addition, SSG reduces tablet hardness, slightly shortens disintegration time, and increases friability and dissolution rate. Prediction of the optimum formulation is allowed from overlaid contour plot that resulted in composition of $2.01 \%$ povidone K-30 and $2.01 \%$ SSG to produce levofloxacin tablet with good tablet properties. 


\section{ACKNOWLEDGEMENT}

The authors would like to acknowledge Novapharin Pharmaceutical Industries for providing levofloxacin and excipients used in this research.

\section{REFERENCES}

Chime, S.A., Browm, S.A., Ugwu, C.E., Agubata, C.O., Obidike, T.C., \&Onunkwo, G.C. 2012. Effect of Binder Type and Concentration on The In Vitro Properties of Alstoniaboonei Tablets. International Journal of Pharmaceutical Science Review and Research. 16(2): 5-9.

Fatmawati, D.A., Widjaja, B., \& Setyawan, D. 2017. Optimization of Levofloxacin Tablet Containing PVP K-30 and Vivasol. Jurnal Sains Farmasi \& Klinis. 4(2): 9-15.

Genç, L. \& Jalvand E. 2008. Preparation and In Vitro Evaluation of Controlled Release Hydrophilic Matrix Tablets of Ketorolac Tromethamine Using Factorial Design. Drug Development and Industrial Pharmacy. 34: 903-910.

Mehta, S., Beer, T.D., Remon, J.P., Vervaet, C. 2011. Effect of Disintegrants on The Properties of Multiparticulate Tablets Comprising Starch Pellets and Excipients Granules. International Journal of Pharmaceutics. 422: 310-317.

Ministry of Health of the Republic of Indonesia 2014. Farmakope Indonesia Edisi $V$. Jakarta: Kementerian Kesehatan RI.

Mohanachandran, P.S., Sindhumol, P.G., \& Kiran, T.S. 2011. Superdisintegrants: an overview. International Journal of Pharmaceutical Science Review and Research. 6(1): 105-109.

Nazir, S.U.R., Nadeem, M., Malik, A., Kamran, Irshad, N., Amer, M., \& Asjad, H.M. 2013. Formulation and In-Vitro Evaluation of Levofloxacin Tablets by Using Different Superdisintegrants. American Journal of Research Communication. 1(4): 193-199.

Prasad M., H.H. \& Duraivel S. (2012). Effect of Different Binders and Super
Disintegrants on Formulation of Glimepiride Immediate Release Tablets by Wet Granulation Method. International Journal of Pharmaceutical and Clinical Research. 4: 44-47.

Rowe, R.C., Sheskey, P.J., Quinn, M.E. (2009). Handbook of Pharmaceutical Excipients $6^{\text {th }} E d$. London: Pharmaceutical Press.

Schmid, K. \&Löbenberg R. 2012. Influence of The Changed USP Specifications on Disintegration Test Performance. Dissolution Technologies. 17(1): 6-10.

Setyawan, D., Widjaja, B., \& Sari, R. 2013. Study on Crystallinity and Compactibility of Binary Mixture of Analgesic Substances With Microcrystalline Cellulose. International Journal of Pharmacy and Pharmaceutical Sciences. 5(3): 784-789.

Shakar, A.A.M., Ibn Razzak, M.S.M., Hossain, M.M., Arif, M.H., \& Reza, M.S. 2012. Effect of Super Disintegrants on Some Physical Attributes and Release Profile of Paracetamol Immediate Release Tablets. Bangladesh Pharmaceutical Journal. 15(1): 89-94.

The USP Convention (The United State Pharmacopeia Convention). 2011. U.S. Pharmacopeia $34^{\text {th }}$ - National Formulary $29^{\text {th }}$. Baltimore: United Book Press, Inc.

Türkmen, Ö., Şenyiğit, Z.A., \& Baloğlu E. 2018. Formulation and Evaluation of Fexofenadine Hydrochloride Orally Disintegrating Tablets For Pedriatic Use. Journal of Drug Delivery Science and Technology. 43: 201-210.

Uhumwangho, M.U., \& Okor, R.S. 2004. Anomalous Effect of Compression Pressure on The Brittle Fracture Tendency of $\alpha$ cellulose Tablets. International Journal of Pharmaceutics. 284: 69-74.

Uhumwangho, M.U., Okor, R.S., Eichie F.E., Abbah, C.M. (2006) Influence of Some Starch Binders on the Brittle Fracture Tendency of Parasetamol Tablets. African Journal of Biotechnology. 5(20): 19501953. 
